\title{
Resolution of system of Volterra integral equations of the first kind by derivation technique and modified decomposition methods
}

\author{
M. A. ALZHRANI, H. O. BAKODAH AND M. AL-MAZMUMY
}

\begin{abstract}
A solution method for various systems of integral equations of the first kind is presented in this paper. The method starts off by transforming the systems via the application of the Leibnitz's derivation technique and then employs three different decomposition methods based on the Standard Adomian decomposition method (SADM) for solutions. To demonstrate the efficiency of the proposed method, some illustrative examples are considered and the obtained results indicate that the approach is indeed of practical interest.
\end{abstract}

Mathematics Subject Classification: 65R20, 65L80, 45G10, 45D05, 41A30

Keywords: Integral equations; Volterra integral equations; Leibnitz's derivation technique; Adomian decomposition method.

\section{INTRODUCTION}

Volterra integral equations and their systems are important class of integral equations that arise in many engineering applications. Such equations have been treated by various numerical and analytical techniques [1-4]. However, despite the fact that analytical solutions are the most desired ones, still numerical methods are considered powerful since they solve many problems that analytical solutions do not exist. Methods like the iterative methods, perturbation methods, series expansion in form of certain functions are the usual numerical or approximation methods. Regarding some numerical techniques for systems of integral equations; system of Fredholm integral equations was numerically solved using the wavelet technique [5,6] while the Standard Adomian decomposition method (SADM) was applied to the various systems of Volterra and Fredholm integral equations, respectively, [7-11]. In addition, further application of the ADM in solving integral equations can be seen in the works of Cherrualt and Seng [12] for certain integral equations of the first kind, Babolian et al. [13] for solving linear and nonlinear systems of Volterra integral equations of the second kind and Biazar et al. [14] for Volterra integral equations of the first kind among others. 
Now that the SADM has been proven to be an efficient semi-analytical method for solving various integral equations since its inception in 1980's [15] leading to undergoing several modifications and improvements by many researchers, see [16-20] and also [21-26]; we therefore aim in this paper to consider the certain modifications of the ADM to study the solution of certain systems of Volterra integral equations of the first kind. To achieve our set goal, we first employ the Leibnitz's derivation technique [1] to transform our system to a canonical system suitable for the ADM based modification procedures. We will also establish some comparative study between the classical ADM and our modification procedures.

\section{VOLTERRA INTEGRAL EQUATIONS}

A system of Volterra integral equations of the first kind can be written as follows:

$$
\int_{a}^{b(x)} k_{i}(x, t) g_{i}\left(u_{1}(t), u_{2}(t), \cdots, u_{n}(t)\right) d t=f_{i}(x), \quad i=1,2, \cdots, n ;
$$

where $f_{i}$ are known functions, $k_{i}(x, t)$ are the kernels of the $i t h$ integral equation, $g_{i}$ are linear or nonlinear functional of the unknown functions $u_{i}$.

We suppose that the system (1) has unique solution. However, the necessary and sufficient conditions for existence and uniqueness of the solution of system (1) could be found in $[21,22]$.

\subsection{Linear case}

The standard form of the system of Volterra integral equations of the first kind is given by

$$
\begin{aligned}
& f_{1}(x)=\int_{0}^{x}\left(K_{1}(x, t) u_{1}(t)+\tilde{K}_{1}(x, t) u_{2}(t)\right) d t \\
& f_{2}(x)=\int_{0}^{x}\left(K_{2}(x, t) u_{1}(t)+\tilde{K}_{2}(x, t) u_{2}(t)\right) d t
\end{aligned}
$$

where the kernels are $K_{i}(x, t)$ and $\tilde{K}_{i}(x, t)$, and the functions $f_{i}(x), i=1,2$ are given realvalued functions, and $u_{i}(x), i=1,2$ are the unknown functions that will be determined. 
Now, differentiating both sides of each equation in (2), and using Leibnitz's derivation technique [1], we obtain

$$
\begin{aligned}
f_{1}^{\prime}(x) & =K_{1}(x, x) u_{1}(x)+\tilde{K}_{1}(x, x) u_{2}(x) \\
& +\int_{0}^{x}\left(K_{1_{x}}(x, t) u_{1}(t)+\tilde{K}_{1_{x}}(x, t) u_{2}(t)\right) d t, \\
f_{2}^{\prime}(x) & =K_{2}(x, x) u_{1}(x)+\tilde{K}_{2}(x, x) u_{2}(x) \\
& +\int_{0}^{x}\left(K_{2_{x}}(x, t) u_{1}(t)+\tilde{K}_{2_{x}}(x, t) u_{2}(t)\right) d t .
\end{aligned}
$$

REMARK 2.1. Three remarks can be made here:

1. If at least one of $K_{i}(x, x)$ and $\tilde{K}_{i}(x, x), i=1,2$ in each of the above equations does not vanish, then the system is reduced to a system of Volterra integral equations of the second kind.

2. If $K_{i}(x, x)=0$ and $\tilde{K}_{i}(x, x)=0, i=1,2$ for any equation, and if $K_{i_{x}}(x, x) \neq 0$ and $K_{i_{x}}(x, x) \neq 0$, then we differentiate again that equation.

3. The functions $f_{i}(x)$ must satisfy specic conditions to guarantee a unique continuous solution for each of the unknown solutions.

\subsection{Nonlinear case}

We will study a specific case of the systems of nonlinear Volterra integral equations of the first kind given by

$$
\begin{aligned}
& f_{1}(x)=\int_{0}^{x}\left(K_{1}(x, t) u_{1}(t)+\tilde{K}_{1}(x, t) F_{1}\left(u_{2}(t)\right)\right) d t, \\
& f_{2}(x)=\int_{0}^{x}\left(K_{2}(x, t) F_{2}\left(u_{1}(t)\right)+\tilde{K}_{2}(x, t) u_{2}(t)\right) d t,
\end{aligned}
$$

where the kernels are $K_{i}(x, t)$ and $\tilde{K}_{i}(x, t)$, and the functions $f_{i}(x)$ are given real-valued functions and $u_{i}(x), i=1,2$ are the unknown functions that will be determined. Recall that the unknown functions $u_{i}(x)$ appear inside the integral sign for the Volterra integral equations of the first kind. We first need to convert this system to a system of nonlinear Volterra integral equation of the second kind. This can be achieved by differentiating both sides of each part of the system. The conversion technique works effectively by using Leibnitz's technique or rule. Differentiating both sides of each equation in (3) 
and using Leibnitz's rule, we obtain

$$
\begin{aligned}
f_{1}^{\prime}(x) & =K_{1}(x, x) u_{1}(x)+\tilde{K}_{1}(x, x) F_{1}\left(u_{2}(x)\right) \\
& +\int_{0}^{x}\left(K_{1_{x}}(x, t) u_{1}(t)+\tilde{K}_{1_{x}}(x, t) F_{1}\left(u_{2}(t)\right)\right) d t, \\
f_{2}^{\prime}(x) & =K_{2}(x, x) F_{2}\left(u_{1}(x)\right)+\tilde{K}_{2}(x, x) u_{2}(x) \\
& +\int_{0}^{x}\left(K_{2_{x}}(x, t) F_{2}\left(u_{1}(t)\right)+\tilde{K}_{2_{x}}(x, t) u_{2}(t)\right) d t . \\
u_{1}(x)= & \frac{f_{1}^{\prime}(x)-\tilde{K}_{1}(x, x) F_{1}\left(u_{2}(x)\right)}{K_{1}(x, x)} \\
- & \frac{1}{K_{1}(x, x)} \int_{0}^{x}\left(K_{1_{x}}(x, t) u_{1}(t)+\tilde{K}_{1_{x}}(x, t) F_{1}\left(u_{2}(t)\right)\right) d t, \\
u_{2}(x)= & \frac{f_{2}^{\prime}(x)-K_{2}(x, x) F_{2}\left(u_{1}(x)\right)}{\tilde{K}_{2}(x, x)} \\
- & \frac{1}{\tilde{K}_{2}(x, x)} \int_{0}^{x}\left(K_{2_{x}}(x, t) F_{2}\left(u_{1}(t)\right)+\tilde{K}_{2_{x}}(x, t) u_{2}(t)\right) d t .
\end{aligned}
$$

It is obvious that the last system is a system of nonlinear Volterra integral equations of the second kind. Notice that the non-homogeneous terms and the kernels have changed to

$$
\begin{aligned}
g_{1}(x) & =\frac{f_{1}^{\prime}(x)-\tilde{K}_{1}(x, x) F_{1}\left(u_{2}(x)\right)}{K_{1}(x, x)}, \\
g_{2}(x) & =\frac{f_{2}^{\prime}(x)-K_{2}(x, x) F_{2}\left(u_{1}(x)\right)}{\tilde{K}_{2}(x, x)}, \\
G_{1}(x, t) & =\frac{K_{1_{x}}(x, t)}{K_{1}(x, x)}, \quad \tilde{G}_{1}(x, t)=\frac{\tilde{K}_{1_{x}}(x, t)}{K_{1}(x, x)}, \\
G_{2}(x, t) & =\frac{K_{2_{x}}(x, t)}{K_{2}(x, x)}, \quad \tilde{G}_{2}(x, t)=\frac{\tilde{K}_{2_{x}}(x, t)}{K_{2}(x, x)} .
\end{aligned}
$$

Then, the form of the system of nonlinear Volterra integral equation of the second kind becomes

$$
\left\{\begin{array}{l}
u_{1}(x)=g_{1}(x)-\int_{0}^{x}\left(G_{1}(x, t) u_{1}(t)+\tilde{G}_{1}(x, t) F_{1}\left(u_{2}(t)\right)\right) d t \\
u_{2}(x)=g_{2}(x)-\int_{0}^{x}\left(G_{2}(x, t) F_{2}\left(u_{1}(t)\right)+\tilde{G}_{2}(x, t) u_{2}(t)\right) d t
\end{array}\right.
$$


which is in the Adomian's canonical form with the following recursive scheme:

$$
\begin{aligned}
u_{1,0}(x) & =g_{1}(x), \\
u_{1, n+1}(x) & =-\int_{0}^{x}\left(G_{1}(x, t) u_{1, n}(t)+\tilde{G}_{1}(x, t) A_{2, n}(t) d t,\right. \\
u_{2,0}(x) & =g_{2}(x) \\
u_{2, n+1}(x) & =-\int_{0}^{x}\left(G_{2}(x, t) A_{1, n}(t)+\tilde{G}_{2}(x, t) u_{2, n}(t)\right), d t
\end{aligned}
$$

where $A_{i, n}, i=1,2$ are the Adomian polynomials given by

$$
A_{n}=\frac{1}{n !} \frac{d^{n}}{d \lambda^{n}}\left[F\left(\sum_{i=0}^{n} \lambda^{i} u_{i}\right)\right]_{\lambda=0}, \quad n=0,1,2, \cdots
$$

\section{SOME MODIFICATIONS OF THE ADM}

\subsection{Reliable modification (M1)}

The standard decomposition method (SADM) by Adomian [15] was modified by Wazwaz [23]. The modification is based on the assumption that the function $g_{i}(x), i=$ 1,2 can be divided into two parts, namely, $g_{i, o}(x)$ and $g_{i, 1}(x)$. Under this assumption we set

$$
g_{i}(x)=g_{i, 0}(x)+g_{i, 1}(x) .
$$

Accordingly, a slight variation was proposed only on the components $u_{i, 0}$ and $u_{i, 1}$. The suggestion was that only the part $g_{0, i}$ be assigned to the zeroth component $u_{i, 0}$; whereas the remaining part $g_{1, i}$ should be combined with the other terms to define $u_{i, 1}$. Consequently the modified recursive relation

$$
\begin{aligned}
u_{1,0}(x) & =g_{1,0}(x), \\
u_{1,1}(x) & =g_{1,1}(x)-\int_{0}^{x}\left(G_{1}(x, t) u_{1,0}(t)+\tilde{G}_{1}(x, t) A_{2,0}(t) d t,\right. \\
u_{1, n+1}(x) & =-\int_{0}^{x}\left(G_{1}(x, t) u_{1, n}(t)+\tilde{G}_{1}(x, t) A_{2, n}(t) d t,\right. \\
u_{2,0}(x) & =g_{2,0}(x), \\
u_{2,1}(x) & =g_{2,1}(x)-\int_{0}^{x}\left(G_{2}(x, t) A_{1,0}(t)+\tilde{G}_{2}(x, t) u_{2,0}(t)\right) d t, \\
u_{2, n+1}(x) & =-\int_{0}^{x}\left(G_{2}(x, t) A_{1, n}(t)+\tilde{G}_{2}(x, t) u_{2, n}(t)\right) d t .
\end{aligned}
$$

The choice of $g_{i, 0}$ such that $u_{i, 0}$ contains the minimal number of terms has a strong influence in accelerating the convergence rate of the solution. This means that the 
success of this method depends mainly on the proper choice of $g_{i, 0}$ and $g_{i, 1}$ and it is mainly trials. The modification demonstrates a rapid convergence of the series solution if compared with the SADM, and it may give the exact solution for nonlinear equations by using two iterations only and without using the so-called Adomian polynomials.

\subsection{New modification (M2)}

The modified decomposition method in (3.1) depends entirely on the proper selection of the functions $g_{0, i}$ and $g_{1, i}$. It appears that trials are the only criteria that can be applied so far. In the new modification by Wazwaz [24] we can replace the process of dividing $g_{i}$ into two components by a series of infinite components. We therefore suggest that $g_{i}$ be expressed in Taylor series

$$
g_{i}(x)=\sum_{n=0}^{\infty} g_{i, n}(x) .
$$

A new recursive relationship is then expressed as

$$
\begin{aligned}
u_{1,0}(x) & =g_{1,0}(x), \\
u_{1, n+1}(x) & =g_{1, n}-\int_{0}^{x}\left(G_{1}(x, t) u_{1, n}(t)+\tilde{G}_{1}(x, t) A_{2, n}(t) d t,\right. \\
u_{2,0}(x) & =g_{2,0}(x) . \\
u_{2, n+1}(x) & =g_{2, n}-\int_{0}^{x}\left(G_{2}(x, t) A_{1, n}(t)+\tilde{G}_{2}(x, t) u_{2, n}(t)\right) d t .
\end{aligned}
$$

It is important to note that if $g_{i}$ consists of one term only, then scheme in (10) reduces to relation (7). Moreover if $g_{i}$ consists of two terms, then relation (10) reduces to the modified relation $(8)$.

\subsection{Restarted Adomian decomposition method (M3)}

The restarted Adomian method (RADM) by Babolian et al. [20] was based on the standard ADM for algebraic equations, see also Sadeghi et al. [25] for the application. The method modified the SADM by a slight variation in $u_{i, 0}$ and $u_{i, 1}$ components thereby accelerating the rate of convergence better than the SADM. 
3.3.1. Description of the method. We introduce the algorithm as the following

(1) Choose small natural numbers $n$ and $m$.

(2) Apply the Adomian method on equations (5) to calculate

$$
\left(u_{1,0}, u_{2,0}\right),\left(u_{1,1}, u_{2,1}\right), \cdots,\left(u_{1, m}, u_{2, m}\right) \text {. }
$$

set

$$
\begin{aligned}
& w_{1}^{1}=u_{1,0}+u_{1,1}+\cdots+u_{1, m}, \\
& w_{2}^{1}=u_{2,0}+u_{2,1}+\cdots+u_{2, m} .
\end{aligned}
$$

(3) Let $z_{1}$ and $z_{2}$ be the proper functions which will be determined next.

For $j=2: n$ do

$$
\begin{gathered}
\left\{\begin{array}{l}
w_{1}^{j-1}=z_{1}, \\
w_{2}^{j-1}=z_{2},
\end{array}\right. \\
\left\{\begin{array}{l}
u_{1,0}=z_{1}, \\
u_{2,0}=z_{2},
\end{array}\right. \\
\left\{\begin{array}{l}
u_{1,1}=g_{1}-z_{1}+A_{1,0}, \\
u_{2,1}=g_{2}-z_{2}+A_{2,0},
\end{array}\right. \\
\left\{\begin{array}{l}
u_{1, m+1}=A_{1, m}, \\
u_{2, m+1}=A_{2, m} .
\end{array}\right.
\end{gathered}
$$

set

$$
\begin{aligned}
& w_{1}^{j}=u_{1,0}+u_{1,1}+\cdots+u_{1, m}, \\
& w_{2}^{j}=u_{2,0}+u_{2,1}+\cdots+u_{2, m} .
\end{aligned}
$$

end. 


\section{Remarks}

(1) $w_{i}^{n}$ can be considered as the approximate solution of Eq. (1).

(2) The Adomian method usually gives sum of some first terms as an approximation of $u_{i}$. Thus, in this algorithm we can update $u_{i, 0}$ in each step with the exception of the terms with large index; so $n$ and $m$ are considered small.

\section{NUMERICAL EXAMPLE}

EXAMPLE 4.1. Consider the following system of linear Volterra integral equations with the exact solutions: $u_{1}(x)=x^{2}$ and $u_{2}(x)=x$,

$$
\begin{gathered}
\int_{0}^{x}\left(\left(1-x^{2}+t^{2}\right) u_{1}(t)-(2 x-t) u_{2}(t)\right) d t=-\frac{1}{3} x^{3}-\frac{2}{15} x^{5}, \\
\int_{0}^{x}\left(\left(x+t^{2}\right) u_{1}(t)+(2-x+t) u_{2}(t)\right) d t=x^{2}-\frac{1}{6} x^{3}+\frac{1}{3} x^{4}+\frac{1}{5} x^{5} .
\end{gathered}
$$

Differentiating the above equations with respect to $x$ we have

$$
\begin{gathered}
u_{1}(x)-x u_{2}(x)-2 \int_{0}^{x}\left(x u_{1}(t)+u_{2}(t)\right) d t=-x^{2}-\frac{2}{3} x^{4}, \\
\left(x+x^{2}\right) u_{1}(x)+2 u_{2}(x)+\int_{0}^{x}\left(u_{1}(t)-u_{2}(t)\right) d t=2 x-\frac{1}{2} x^{2}+\frac{4}{3} x^{3}+x^{4} .
\end{gathered}
$$

Or

$$
\begin{gathered}
u_{1}(x)=-x^{2}-\frac{2}{3} x^{4}+x u_{2}(x)+2 \int_{0}^{x}\left(x u_{1}(t)+u_{2}(t)\right) d t \\
u_{2}(x)=x-\frac{1}{4} x^{2}+\frac{2}{3} x^{3}+\frac{1}{2} x^{4}-\frac{1}{2}\left(x+x^{2}\right) u_{1}(x)-\frac{1}{2} \int_{0}^{x}\left(u_{1}(t)-u_{2}(t)\right) d t .
\end{gathered}
$$

Thus, SADM offers the following recursive scheme

$$
\begin{gathered}
u_{1,0}(x)=-x^{2}-\frac{2}{3} x^{4}, \\
u_{2,0}(x)=x-\frac{1}{4} x^{2}+\frac{2}{3} x^{3}+\frac{1}{2} x^{4}, \\
u_{1, n+1}(x)=x u_{2, n}(x)+2 \int_{0}^{x}\left(x u_{1, n}(t)+u_{2, n}(t)\right) d t, \\
u_{2, n+1}(x)=-\frac{1}{2}\left(x+x^{2}\right) u_{1, n}(x)-\frac{1}{2} \int_{0}^{x}\left(u_{1, n}(t)-u_{2, n}(t)\right) d, \quad n=0,1,2, \cdots
\end{gathered}
$$

\section{(i) Relible Modification (M1)}

The M1 methods gives the following recursive scheme from our problem as follows

$$
\begin{gathered}
u_{1,0}(x)=-\frac{2}{3} x^{4}, \\
u_{2,0}(x)=x,
\end{gathered}
$$




$$
\begin{gathered}
u_{1,1}(x)=-x^{2}+x u_{2,0}(x)+2 \int_{0}^{x}\left(x u_{1,0}(t)+u_{2,0}(t)\right) d t \\
u_{2,1}(x)=-\frac{1}{4} x^{2}+\frac{2}{3} x^{3}+\frac{1}{2} x^{4}-\frac{1}{2}\left(x+x^{2}\right) u_{1,0}(x)-\frac{1}{2} \int_{0}^{x}\left(u_{1,0}(t)-u_{2,0}(t)\right) d t \\
u_{1, n+1}(x)=x u_{2, n}(x)+2 \int_{0}^{x}\left(x u_{1, n}(t)+u_{2, n}(t)\right) d t, \\
u_{2, n+1}(x)=-\frac{1}{2}\left(x+x^{2}\right) u_{1, n}(x)-\frac{1}{2} \int_{0}^{x}\left(u_{1, n}(t)-u_{2, n}(t)\right) d t, \quad n=1,2, \cdots
\end{gathered}
$$

\section{(ii) Restarted ADM (M3)}

Considering the small indexes $n$ and $m$, say $n=3, m=4$;

step1:

$$
\begin{aligned}
& \left\{\begin{array}{l}
u_{1,0}(x)=-x^{2}-\frac{2}{3} x^{4} \\
u_{2,0}(x)=x-\frac{1}{4} x^{2}+\frac{2}{3} x^{3}+\frac{1}{2} x^{4}, \\
u_{1, n+1}(x)=x u_{2, n}(x)+2 \int_{0}^{x}\left(x u_{1, n}(t)+u_{2, n}(t)\right) d t \\
u_{2, n+1}(x)=-\frac{1}{2}\left(x+x^{2}\right) u_{1, n}(x)-\frac{1}{2} \int_{0}^{x}\left(u_{1, n}(t)-u_{2, n}(t)\right) d t, n=0,1,2, \cdots
\end{array}\right. \\
& \qquad \begin{array}{l}
u_{1,1}(x)=2 x^{2}-\frac{5}{12} x^{3}+\frac{1}{3} x^{4}+\frac{7}{10} x^{5}-\frac{4}{15} x^{6}, \\
u_{2,1}(x)=\frac{1}{4} x^{2}+\frac{5}{8} x^{3}+\frac{7}{12} x^{4}+\frac{9}{20} x^{5}+\frac{1}{3} x^{6}, \\
u_{1,2}(x)=\frac{5}{12} x^{3}+\frac{109}{48} x^{4}+\frac{73}{120} x^{5}+\frac{11}{15} x^{6}+\cdots \\
u_{2,2}(x)=-\frac{31}{24} x^{3}-\frac{127}{192} x^{4}+\frac{1}{15} x^{5}-\frac{43}{80} x^{6}+\cdots \\
u_{1,3}(x)=-\frac{31}{16} x^{4}-\frac{689}{960} x^{5}+\frac{359}{360} x^{6}+\cdots \\
u_{2,3}(x)=-\frac{27}{64}-\frac{3143}{1920} x^{5}-\frac{1069}{720} x^{6}+\cdots
\end{array} \\
& w_{1}^{1}=u_{1,0}+u_{1,1}+u_{1,2}+u_{1,3}=x^{2}+\frac{189}{320} x^{5}+\frac{527}{360} x^{6}+\cdots \\
& w_{2}^{1}=u_{2,0}+u_{2,1}+u_{2,2}+g_{2,3}=x-\frac{717}{640} x^{5}-\frac{76}{45} x^{6}+\cdots
\end{aligned}
$$


step2:

$$
\left\{\begin{array}{l}
u_{1,0}(x)=x^{2}+\frac{189}{320} x^{5}+\frac{527}{360} x^{6}+\cdots \\
u_{2,0}(x)=x-\frac{717}{640} x^{5}-\frac{76}{45} x^{6}+\cdots \\
u_{1,1}(x)=\left(x^{2}-\frac{2}{3} x^{4}\right)-\left(x^{2}+\frac{189}{320} x^{5}+\frac{527}{360} x^{6}+\cdots\right)+x u_{2,0}(x)+2 \int_{0}^{x}\left(x u_{1,0}(t)+u_{2,0}(t)\right) d t, \\
u_{2,1}(x)=\left(x-\frac{1}{4} x^{2}+\frac{2}{3} x^{3}+\frac{1}{2} x^{4}\right)-\left(x-\frac{717}{640} x^{5}-\frac{76}{45} x^{6}+\cdots\right)-\frac{1}{2}\left(x+x^{2}\right) u_{1,0}(x) \\
-\frac{1}{2} \int_{0}^{x}\left(u_{1,0}(t)-u_{2,0}(t)\right) d t \\
u_{1, n+1}(x)=x u_{2, n}(x)+2 \int_{0}^{x}\left(x u_{1, n}(t)+u_{2, n}(t)\right) d t \\
u_{2, n+1}(x)=-\frac{1}{2}\left(x+x^{2}\right) u_{1, n}(x)-\frac{1}{2} \int_{0}^{x}\left(u_{1, n}(t)-u_{2, n}(t)\right) d t, n=1,2, \cdots
\end{array}\right.
$$

$$
\left\{\begin{array}{l}
u_{1,1}(x)=-\frac{189}{320} x^{5}-\frac{4259}{1440} x^{6}-\frac{43307}{20160} x^{7}+\cdots \\
u_{2,1}(x)=\frac{717}{640} x^{5}+\frac{28823}{23040} x^{6}-\frac{4261}{13440} x^{7}+\cdots \\
u_{1,2}(x)=\frac{239}{160} x^{6}+\frac{5059}{3584} x^{7}-\frac{200203}{161280} x^{8}+\cdots \\
u_{2,2}(x)=\frac{1121}{2560} x^{6}+\frac{74359}{35840} x^{7}+\frac{1720759}{645120} x^{8}+\cdots \\
u_{1,3}(x)=\frac{10089}{17920} x^{7}+\frac{432979}{143360} x^{8}+\cdots \\
u_{2,3}(x)=-\frac{29471}{35840} x^{7}-\frac{809239}{573440} x^{8}+\cdots \\
w_{1}^{2}=x^{2}+\frac{29471}{2872} x^{8}-\frac{1139}{560} x^{9}+\cdots \\
w_{2}^{2}=x+\frac{211073}{573440} x^{8}-\frac{213207}{143360} x^{9}+\cdots
\end{array}\right.
$$

step3:

$$
\begin{gathered}
w_{1}^{3}=x^{2}-\frac{1487022307}{851558400} x^{12}-\frac{81990701}{252313600} x^{11}+\cdots \\
w_{2}^{3}=x+\frac{519896129}{425779200} x^{12}+\frac{175553087}{504627200} x^{11}+\cdots
\end{gathered}
$$

step4:

$$
\begin{gathered}
w_{1}^{3}=x^{2}-\frac{162739797083}{166985728000} x^{15}-\frac{66509498791}{275526451200} x^{14}+\cdots \\
w_{2}^{4}=x-\frac{28883455355929}{33063174144000} x^{15}-\frac{100436326331}{801531494400} x^{14}+\cdots
\end{gathered}
$$

We therefore establish the comparisons between the exact solution and the solutions obtained by SADM, M1 and M3 methods for $u_{1}(x)$ and $u_{2}(x)$ and reported in Tables 1 and 2, and Figures 1 and 2, respectively. 


\begin{tabular}{|c|c|c|c|c|c|c|c|}
\hline$x$ & $u_{1}(x)$ & SADM & error & M1 & error & M3 & error \\
\hline 0.1 & 0.01 & 0.009998 & 0.000002 & 0.009999 & 0.000001 & 0.01 & 0 \\
\hline 0.2 & 0.04 & 0.039877 & 0.000123 & 0.039935 & 0.000065 & 0.04 & 0 \\
\hline 0.3 & 0.09 & 0.088414 & 0.001586 & 0.089162 & 0.000838 & 0.09 & 0 \\
\hline 0.4 & 0.16 & 0.149935 & 0.010065 & 0.154715 & 0.005285 & 0.159998 & 0.000002 \\
\hline 0.5 & 0.25 & 0.206619 & 0.043381 & 0.227417 & 0.022583 & 0.249929 & 0.000071 \\
\hline
\end{tabular}

Table I: Comparison between exact solution $u_{1}(x)$ and approximate solutions using SADM, M1 and M3 methods

\begin{tabular}{|c|c|c|c|c|c|c|c|}
\hline$x$ & $u_{2}(x)$ & SADM & error & M1 & error & M3 & error \\
\hline 0.1 & 0.1 & 0.099999 & 0.000001 & 0.1 & 0 & 0.1 & 0 \\
\hline 0.2 & 0.2 & 0.199954 & 0.000046 & 0.199981 & 0.000019 & 0.2 & 0 \\
\hline 0.3 & 0.3 & 0.299346 & 0.000654 & 0.299690 & 0.000310 & 0.3 & 0 \\
\hline 0.4 & 0.4 & 0.395531 & 0.004469 & 0.397601 & 0.002399 & 0.399997 & 0.000003 \\
\hline 0.5 & 0.5 & 0.479537 & 0.020463 & 0.487737 & 0.012263 & 0.499886 & 0.000114 \\
\hline
\end{tabular}

Table II: Comparison between exact solution $u_{2}(x)$ and approximate solutions using SADM, M1 and M3 methods

Figure1: The Exact solution $\mathrm{u}_{-} 1(\mathrm{x})$ and Approximate solution using methods

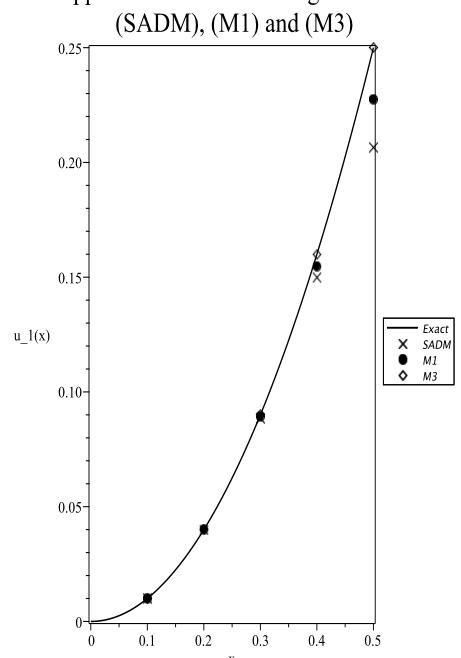

Figure2: The Exact solution u_2(x) and Approximate solution using methods (SADM), (M1) and (M3)

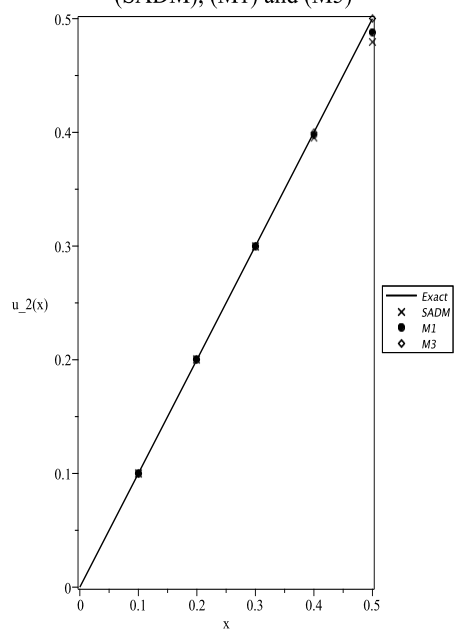

EXAMPLE 4.2. Consider the following system of nonlinear Volterra integral equations given the exact solutions: $u_{1}(x)=x+e^{x}$, and $u_{2}(x)=x-e^{x}$;

$$
\begin{gathered}
\int_{0}^{x}\left(u_{1}(t)+(x-t) u_{1}(t) u_{2}(t)\right) d t=-\frac{3}{4}+\frac{1}{2} x+\frac{1}{2} x^{2}+\frac{1}{12} x^{4}+e^{x}-\frac{1}{4} e^{2 x}, \\
\int_{0}^{x}\left(u_{1}(t)+(x-t) u_{1}(t) u_{2}(t)\right) d t=\frac{5}{4}+\frac{1}{2} x+\frac{1}{2} x^{2}+\frac{1}{12} x^{4}-e^{x}-\frac{1}{4} e^{2 x} .
\end{gathered}
$$


Differentiating the above equation with respect to $x$ we get:

$$
\begin{aligned}
& u_{1}(x)+\int_{0}^{x} u_{1}(t) u_{2}(t) d t=\frac{1}{2}+x+\frac{1}{3} x^{3}+e^{x}-\frac{1}{2} e^{2 x} \\
& u_{2}(x)+\int_{0}^{x} u_{1}(t) u_{2}(t) d t=\frac{1}{2}+x+\frac{1}{3} x^{3}-e^{x}-\frac{1}{2} e^{2 x} .
\end{aligned}
$$

Or

$$
\begin{aligned}
& u_{1}(x)=\frac{1}{2}+x+\frac{1}{3} x^{3}+e^{x}-\frac{1}{2} e^{2 x}-\int_{0}^{x} u_{1}(t) u_{2}(t) d t \\
& u_{2}(x)=\frac{1}{2}+x+\frac{1}{3} x^{3}-e^{x}-\frac{1}{2} e^{2 x}-\int_{0}^{x} u_{1}(t) u_{2}(t) d t
\end{aligned}
$$

Applying the SADM to above system yields the following recursive scheme:

$$
\begin{gathered}
u_{1,0}(x)=\frac{1}{2}+x+\frac{1}{3} x^{3}+e^{x}-\frac{1}{2} e^{2 x}, \\
u_{2,0}(x)=\frac{1}{2}+x+\frac{1}{3} x^{3}-e^{x}-\frac{1}{2} e^{2 x}, \\
u_{1, n+1}(x)=-\int_{0}^{x} A_{n}(t) d t, \\
u_{2, n+1}(x)=-\int_{0}^{x} A_{n}(t) d t, \quad n=0,1,2, \cdots
\end{gathered}
$$

where $A_{n}$ are the Adomian polynomials given by:

$$
\begin{aligned}
& A_{0}(t)=u_{1,0}(t) u_{2,0}(t), \\
& A_{1}(t)=u_{1,0}(t) u_{2,1}(t)+u_{1,1}(t) u_{2,0}(t), \\
& A_{2}(t)=u_{1,0}(t) u_{2,2}(t)+u_{1,2}(t) u_{2,0}(t)+u_{1,1}(t) u_{2,1}(t),
\end{aligned}
$$

and so on.

\section{(i) Relible Modification (M1)}

On using M1, the following recursive scheme to the problem is obtained:

$$
\begin{gathered}
u_{1,0}(x)=x+e^{x} \\
u_{2,0}(x)=x-e^{x} \\
u_{1,1}(x)=\frac{1}{2}+\frac{1}{3} x^{3}-\frac{1}{2} e^{2 x}-\int_{0}^{x} A_{0}(t) d t \\
u_{2,1}(x)=\frac{1}{2}+\frac{1}{3} x^{3}-\frac{1}{2} e^{2 x}-\int_{0}^{x} A_{0}(t) d t \\
u_{1, n+1}(x)=-\int_{0}^{x} A_{n}(t) d t, \\
u_{2, n+1}(x)=-\int_{0}^{x} A_{n}(t) d t, \quad n=1,2, \cdots
\end{gathered}
$$




\section{(ii) New Modification (M2)}

Using M2 method, we first set the Taylor's expansion for $f_{i}(x)$ as

$$
\begin{gathered}
f_{1}(x)=1+x-\frac{1}{2} x^{2}-\frac{1}{6} x^{3}+o[x]^{4} \\
f_{2}(x)=-1-x-\frac{3}{2} x^{2}-\frac{1}{2} x^{3}+o[x]^{4}
\end{gathered}
$$

Thus, we get the following recursive relation from (10);

$$
\begin{gathered}
u_{1,0}(x)=1, \\
u_{2,0}(x)=-1, \\
u_{1,1}(x)=x-\int_{0}^{x} A_{0}(t) d t=2 x, \\
u_{2,1}(x)=-x-\int_{0}^{x} A_{0}(t) d t=0, \\
u_{1,2}(x)=-\frac{1}{2} x^{2}-\int_{0}^{x} A_{1}(t) d t=\frac{1}{2} x^{2}, \\
u_{2,2}(x)=-\frac{3}{2} x^{2}-\int_{0}^{x} A_{1}(t) d t=-\frac{1}{2} x^{2}, \\
u_{1,3}(x)=-\frac{1}{6} x^{3}-\int_{0}^{x} A_{2}(t) d t=\frac{1}{6} x^{3}, \\
u_{2,3}(x)=-\frac{1}{2} x^{3}-\int_{0}^{x} A_{2}(t) d t=-\frac{1}{6} x^{3},
\end{gathered}
$$

and so on. We establish the comparisons between the exact solution and the solutions obtained by M1 and M2 methods for $u_{1}(x)$ and $u_{2}(x)$ and reported in Tables 3 and 4, and Figures 3 and 4, respectively.

\begin{tabular}{|c|c|c|c|c|c|c|c|}
\hline$x$ & $u_{1}(x)$ & SADM & error & M1 & error & M2 & error \\
\hline 0.1 & 1.205171 & 1.205169 & 0.000002 & 1.205171 & 0 & 1.205167 & 0.000004 \\
\hline 0.2 & 1.421403 & 1.421337 & 0.000065 & 1.421403 & 0 & 1.421333 & 0.000069 \\
\hline 0.3 & 1.649859 & 1.649245 & 0.000614 & 1.649859 & 0 & 1.649462 & 0.000359 \\
\hline 0.4 & 1.891825 & 1.888585 & 0.003239 & 1.891825 & 0 & 1.890667 & 0.001158 \\
\hline 0.5 & 2.148721 & 2.136289 & 0.012432 & 2.148721 & 0 & 2.145833 & 0.002888 \\
\hline
\end{tabular}

Table III: Comparison between exact solution $u_{1}(x)$ and approximate solutions using SADM, M1 and M2 methods 


\begin{tabular}{|c|c|c|c|c|c|c|c|}
\hline$x$ & $u_{2}(x)$ & SADM & error & M1 & error & M2 & error \\
\hline 0.1 & -1.005171 & -1.005173 & 0.000002 & -1.005171 & 0 & -1.005167 & 0.000004 \\
\hline 0.2 & -1.021403 & -1.021468 & 0.000066 & -1.021403 & 0 & -1.021333 & 0.000064 \\
\hline 0.3 & -1.049859 & -1.050473 & 0.000614 & -1.049859 & 0 & -1.049500 & 0.000359 \\
\hline 0.4 & -1.091825 & -1.095064 & 0.003239 & -1.091825 & 0 & -1.090667 & 0.001158 \\
\hline 0.5 & -1.148721 & -1.161153 & 0.012432 & -1.148721 & 0 & -1.145833 & 0.002888 \\
\hline
\end{tabular}

Table IV: Comparison between exact solution $u_{2}(x)$ and approximate solutions using SADM, M1 and M2 methods

Figure3: The Exact solution $\mathrm{u}_{-} 1(\mathrm{x})$ and Approximate solution using methods (SADM), (M1) and (M2)

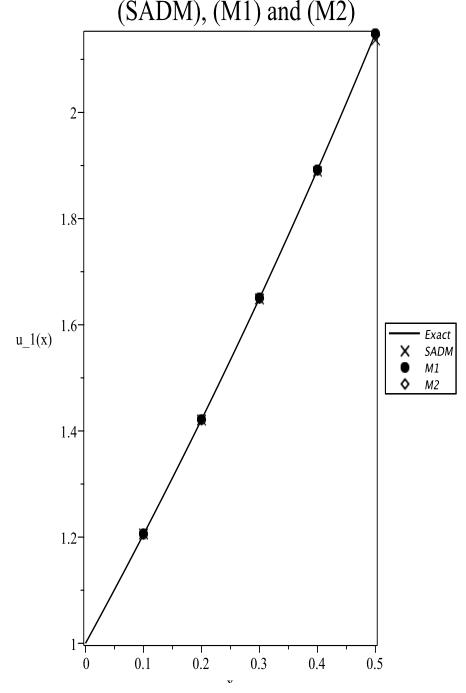

Figure4: The Exact solution u_2(x) and Approximate solution using methods

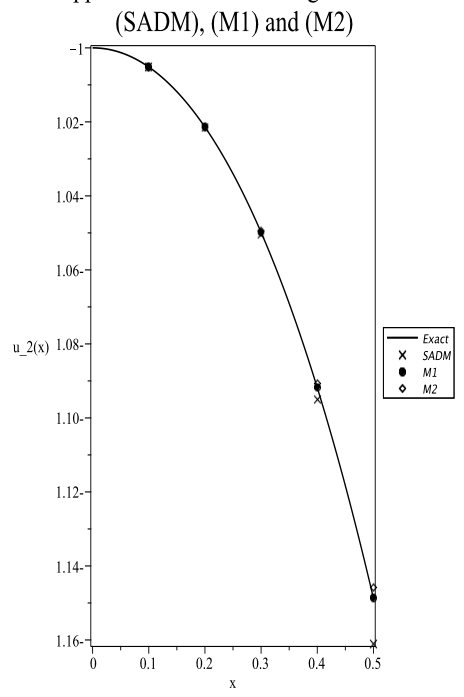

\section{CONCLUSIONS}

In conclusion, we have presented three recursive schemes based on the modifications of the Standard Adomian decomposition method (SADM) for solving system of Volterra integral equations of the first kind. However in doing that, we successfully utilized the Leibniz' derivation technique to transform the integral equations to the conical forms where Adomian technique is applicable. We further applied the presented schemes to some test problems and found remarkable approximates solutions as reported in the given tables and graphs. The computations associated with the test problems were performed using a Maple. 


\section{REFERENCES}

K. E. Atkinson, The Numerical Solution of Integral Equations of the Second Kind, Cambridge University Press, Cambridge, (1997).

R. Kress, Linear Integral Equation. New York: Springer-Verlag (1998).

P.K. Kythe and P. Puri, Computational methods for linear integral equations. Birkhauser Verlag, Springer, Boston, (2002).

P. Linz, Analytical and Numerical Methods for Volterra Equations, SIAM, Philadelphia, (1985).

B. Alpert, G. Beylkin, R. Coifman, V. Rokhlin, Waveletlike bases for the fast solution of second-kind integral equations, SIAM Journal on Scientific Computing, 14 (1993), 159-184.

W.L. Golik, Wavelet packets for fast solution of electromagnetic integral equations, IEEE Trans. Ant. prop. 46 (1998), 618-624.

E. Babolian, J. Biazar, and A.R. Vahidi, The decomposition method applied to system of Fredholm integral equations of the second kind, Appl. Math. Comput. 148 (2004), 443-452.

J. Biazar, E. Babolian, R. Islam, Solution of the system of Volterra integral equations of the first kind by Adomian decomposition method, Appl. Math. Comput. 139 (2003), 249-258.

H.O. Bakodah, Some modifications of Adomian decomposition method applied to nonlinear system of Fredholm integral equations of the second kind, International Journal of Contemporary Mathematical Sciences, 7(19), (2012), 929-942.

H.O. Bakodah. A new modification of the Laplace Adomian decomposition method for system of integral equations, Journal of American Science, 8(10), (2012), 241-246.

H.O. Bakodah, A comparison study between a Chebyshev collocation method and the Adomian decomposition method for solving linear system of Fredholm integral equations of the second kind, Journal of King Abdulaziz University:science, 24(1), (2012), 49-59.

Y. Cherruault, V. Seng, The resolution of non-linear integral equations of the rst kind using the decompositional Method of Adomian, Kebernetes, 26(2), (1997), 198-206.

E. Babolian, J. Biazar, Solution of a System of Nonlinear Volterra Integral Equations of the Second kind, Far East J. Math. Sci. 2(6), (2000), 935-945.

J. Biazar, E. Babolian, R. Islam, Solution of a system of Volterra integral equations of the rst kind by Adomian method, Applied Mathematics and Computation, 139, (2003), 249-258.

G. Adomian, Solving Frontier Problems of Physics: The Decomposition Method, Kluwer Academic Publishers, Boston, MA, (1994).

A.M. Wazwaz, A reliable modication of the Adomian decomposition method, Appl. Math. Comput., 102 (1999), 77-86.

A.M. Wazwaz and S. M. El-Sayed,A new modification of the Adomian decomposition method for linear and nonlinear operators, Applied Mathematics and Computation,122(3),(2001), 393-405.

X. Lou, A two-step Adomian decomposition method, Appl. Math. Comput., 170, (2005), 570-583.

H. Jafari, V. Daftardar-Gejji, Revised Adomian decomposition method for solving a system of nonlinear equations. Appl. Math. Comput., 175, (2006), 1-7.

E. Babolian, S. Javadi, Restarted Adomian method for algebraic equations, Appl. Math. Comput., 146, (2003), 533-541. 
L. M. Delves and J.L. Mohamed, Computational method for integral equations, Cambridge university press, New York, NY. (1985).

R. F. Churchous, Numerical Methods: Handbook of Applicable Mathematics, Vol III, (1981), spriner, New York, NY.

A.M. Wazwaz, A comparison study between the modified decomposition method and the traditional methods for solving nonlinear integral equations, Applied Mathematics and Computation, 181 (2006), 1703 1712.

A.M. Wazwaz,The modified decomposition method for analytic treatment of nonlinear integral equations and systems of nonlinear integral equations, International Journal of Computer Mathematics, 82(9), (2005), 1107-1115.

H.S. Goghary, Sh. Javadi and E. Babolian, Restarted Adomian method for system of nonlinear Volterra integral equations, Appl. Math. Comput. 161 (2005), 745-751.

R.I. Nuruddeen, L. Muhammad, A.M. Nass and T.A. Sulaiman, A review of the integral transforms-based decomposition methods and their applications in solving nonlinear PDEs, Palestine J. Math. 7, (2018), 262-280.

M. A. Alzhrani

Department of Mathematics, Faculty of Science,

King Abdul-Aziz University,

Jeddah, Saudi Arabia.

H. O. Bakodah

Mathematics Department, Faculty of Science, University of Jeddah, P.O. Box 80327,

Jeddah 21589, Saudi Arabia.

email: h.o.bakodah@gmail.com

M. Al-Mazmumy

Mathematics Department, Faculty of Science, University of Jeddah, P.O. Box 80327, Jeddah 21589, Saudi Arabia.

Corresponding author: mome0505@hotmail.com 